\title{
AUTOMATED IDENTIFICATION OF MOSQUITO (DIPTERA: CULICIDAE) WINGBEAT WAVEFORM BY ARTIFICIAL NEURAL NETWORK ${ }^{* *}$
}

\author{
Zhenyu $\mathrm{Li}^{1}{ }^{1,2}$, Zuji Zhou ${ }^{1}$, Zuorui Shen ${ }^{2 *}$ and Qing Yao ${ }^{2}$ \\ 1 College of Agronomy, Sichuan Agricultural University, No.23 Xinkang,Ya'an,Sichuan
}

2 College of Agronomy and Biotechnology,China Agricultural University,No.2 Westroad Yuanmingyuan, Beijing, China

Abstract: The wingbeat waveforms of Aedes albopictus (Skuse), A. aegypti (Linnaeus), Culex pipiens pallens (Coquillett), C. pipiens quinquefasciatus Say, and C. pipiens molestus Forskal were recorded by a photosensor and WfRer system. Wingbeat frequencies were extracted from the wingbeat waveform. Back Propagation artificial neural network classifiers were built to identify automatically the species of mosquitoes by wingbeat waveform, wingbeat frequencies, and combining them as input variables. The most accurate classifier tested was an artificial neural network by wingbeat frequencies as input variable. The accuracy was average $72.67 \%$ and highest $89.00 \%$. It also demonstrated that it was possible to identify automatically the species of mosquitoes. Meanwhile, it probably depended on the characteristic of frequencies for identifying each other among different mosquitoes.

Key words: mosquito, wingbeat frequency, artificial neural network, automated identification

\footnotetext{
* Communication author, email address: ipmist@cau.edu.cn

** National Priority Program of Innovative Technology (863) No. 2002AA243031 and 2003AA2090.
} 


\section{INTRODUCTION}

With the rapid development of entomological taxonomy, artificial neural network theory and wingbeat frequencies of flying insects, which had been used to identify the species of insect, were more efficient, convenient and prompt than traditional morphology ways which needed necessary expert and special apparatus. Many animals, including grasshopper (David, 2004), spiders (David Reby et al., 1997), and bat (Parsons \& Jones 2000) etc., had been identified automatically by artificial neural network. Wingbeat frequencies of flying insects might play significant role in insect behavior. The wingbeat frequencies were complicated, which were produced by wing beat, and formed all kinds of wingbeat waveforms. Lots of them what were caused by physical characteristics of the wings and their motion through the air provide much complex information, which might be used to communicate among insects (Williams et al. 1950). Peter \& Robert (1979) measured the wingbeat frequencies of two species of mosquitoes and found median value were within $5 \mathrm{~Hz}$ of each other under the same condition. Different species of mosquitoes could mate (Taylor \& Brust 1974), which proved the role of sounds in mosquito behavior. It was possible to communicate information between the female and the male through identifying fundamental wingbeat frequency of each other though the wingbeat frequencies of some species of mosquitoes were partly overlapped (Christophers $1960 \&$ Clements 1963). It would be remarkable to identify the wingbeat frequencies of species of mosquitoes by artificial neural network, which had the ability of simulating human brain to distinguish difference of wingbeat frequencies between different mosquitoes with amount of nerve cells. After many years quantifying the wingbeat frequencies by processing the flying sound, which was recorded in the recorder, a photosensor what was designed to record the wingbeat waveform of two species of mosquitoes (Moore ea al. 1986) was based on the discovery that the wings of individual insects could reflect the transient waveform by an exposed photocell (Richards 1955). An optical sensor and data acquisition software for automated monitoring of flying insects were developed (Moore 1998), which could automate to record the transient waveform, and had been put into practice of automated identification. In this paper, it was by building and training an artificial neural network that the wingbeat waveforms of mosquitoes were identified in order to classify species of mosquitoes. 


\section{MATERIAL AND METHODS}

\subsection{Mosquitoes}

Five species of mosquitoes including Aedes albopictus (Skuse), A. aegypti (Linnaeus), Culex pipiens pallens Coquillett), C. pipiens quinquefasciatus Say and C. pipiens molestus Forskal were provided by Institute of Microbiology and Epidemiology, Academy of Military Medical Sciences, China. All mosquitoes fed 5\% glucose solution and maintained in the laboratory at $24{ }^{\circ} \mathrm{C}$ for a maximum of 2 days. Due to different wingbeat frequencies of male and female in the same species, female and male were distinguished.

\subsection{Recording of Wingbeat Waveforms}

For each recording session, only one mosquito was transferred into a transparent plastic jar measuring $17 \mathrm{~cm} \times 14 \mathrm{~cm} \times 14 \mathrm{~cm}$ and fitted with a fine muslin top (Fig. 1). Females and males were recorded separately in each colony. The jar was covered black cloth for shield the flashes of $\mathrm{AC}$ power room light and placed between a DC power lamp and an optical sensor (Qubit Systems, Kingston, Ontario, Canada 2001). The optical sensor was connected to the microphone input port of a 16-bit sound card installed in the computer (Pentium IV 2.0G, $256 \mathrm{MB}$ RAM). During recording waveforms, temperature was maintained in $24^{\circ} \mathrm{C}\left( \pm 0.5^{\circ} \mathrm{C}\right)$ in the jar. A transient waveform recorder program (WfRec, Qubit Systems 2001) was used to sample data and the sampling rate was $4,000 \mathrm{~Hz}$. Wingbeat waveform recordings were separated into two groups. The first group (training data) was used for training artificial neural network classifiers. The second group (evaluating data) was used only for evaluating the classifiers.

\subsection{Data processing}

The raw data recorded by WfRec were processed and devided into three groups of variables. Fist, wingbeat waveform time series $\mathrm{x} 1, \mathrm{x} 2 \ldots \ldots \times 300$, which contains the signal amplitude at discrete time steps of $1 / 4000 \mathrm{~s}$. Second, the spectral information of wingbeat waveforms $f 1, f 2, f 3$, and $f 4$. The $\mathrm{fl}$ is the fundamental frequency that was wingbeat frequency, and $\mathrm{f} 2, \mathrm{f} 3$, and $\mathrm{f} 4$ represented the double frequency, triple frequency, and fourfold frequency harmonics within frequency band of each waveform (Fig. 3). 
Third, data included $\mathrm{x} 1, \mathrm{x} 2, \ldots, \mathrm{x} 300$ and $\mathrm{f1}, \mathrm{f} 2, \mathrm{f} 3, \mathrm{f} 4$. All data were normalized so that the values of each variable ranged between zero and one.

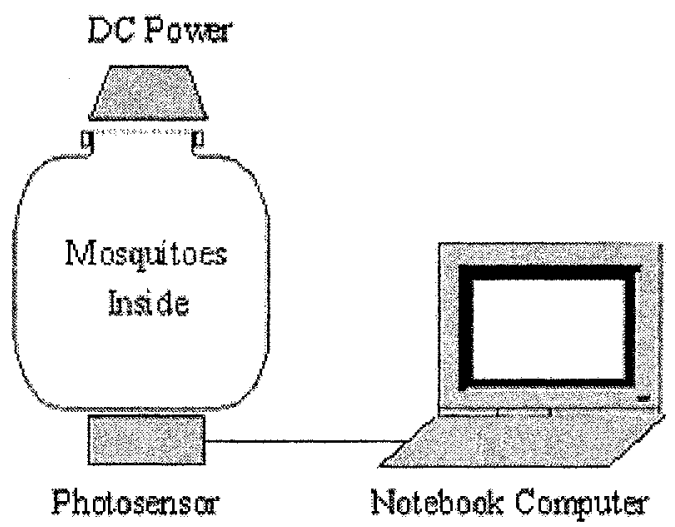

Figure.l Apparatus for digital recording of insect wingbeat waveforms

\subsection{Artificial neural network automated classification}

Three BP artificial neural networks were built respectively to accept input data in the form of a wingbeat waveform time series, signature harmonics and their combination. These networks had all 100 input neurons and 9 output neurons, one output for each mosquito species. There were also five hidden layers with 10 neurons each layer. During training, the network was presented with three groups of variables as input respectively and a nine-digit vector consisting of a 1 and eight zeros to indicate the species of mosquito that produced the signature (e.g., male Aedes albopictus represented by 1000000000 ). Convergence goal was 0.0001 , value of logic judging is 0.5 . After training, evaluating data were presented to the artificial neural network having been trained. Each output vector was classified as one species of mosquito.

\section{RESULTS}

Fig.2 showed the result of recording wingbeat waveform. According to the figure, automated classification waveforms of each species of mosquito were so similar sine wave that it was difficult to identify individual by waveform data alone. As a result of identification in Table1, only accurate $39 \%$ met by artificial neural network, which built with wingbeat waveform 
signature. The most accurate classification model tested was an artificial neural network that was constructed by using variable of wingbeat frequency with mean $72.67 \%$ and highest $89 \%$, ranging from $89 \%$ for male Aedes aegypti (Linnaeus) down to 33\% for female Aedes albopictus (Skuse). Male Aedes aegypti (Linnaeus) was low, which attributed to not evident fourfold frequency. If wingbeat waveform and wingbeat frequency were used as characteristic value of recognition to build artificial neural network, the accuracy had a drop.

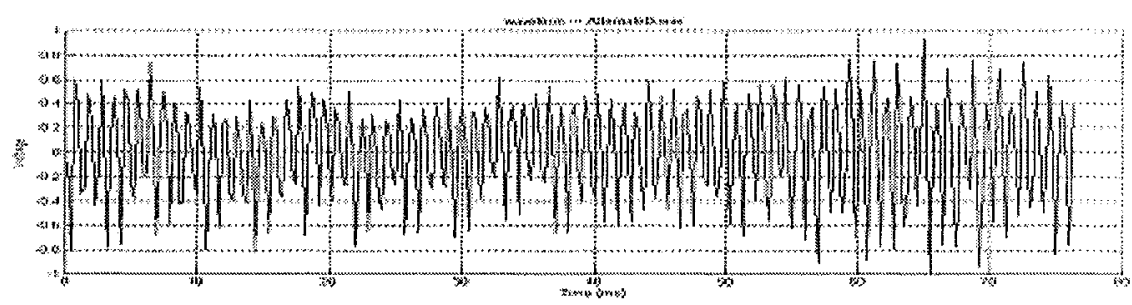

Figure2. Wingbeat waveforms of mosquitoes. Digital processing for calculation of a waveform signature starts with a time series of data point sampled at 4,000 samples per second.

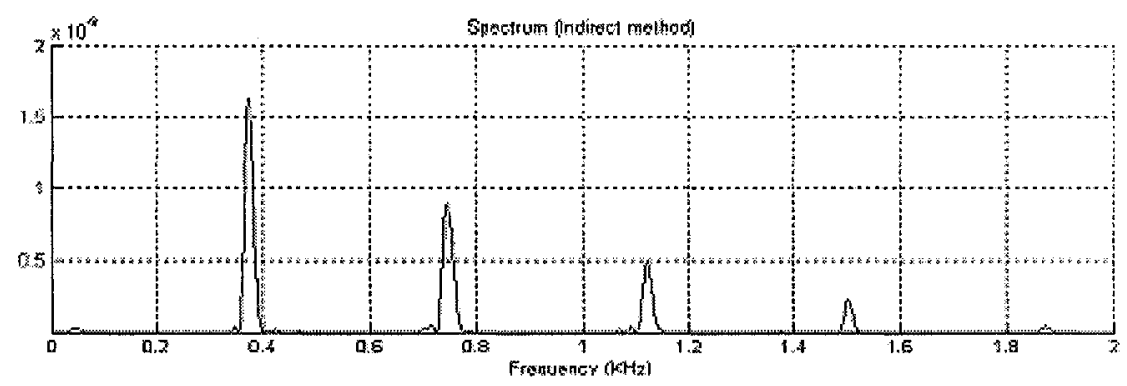

Figure 3. Wingbeat frequencies of mosquitoes. A frequency spectrum was produced by Fourier transform and contained a harmonic series 
Table1.Automated identification by artificial neural network

\begin{tabular}{|c|c|c|c|c|}
\hline & & \multicolumn{3}{|c|}{ Characteristic vector of inputting ANN } \\
\hline \multicolumn{2}{|c|}{ Species of mosquitoes } & $\begin{array}{c}\text { Data of } \\
\text { wingbeat } \\
\text { waveform }\end{array}$ & $\begin{array}{l}\text { Data of wingbeat } \\
\text { frequencies }\end{array}$ & $\begin{array}{l}\text { Data of wingbeat } \\
\text { frequencies and } \\
\text { wingbeat waveform }\end{array}$ \\
\hline \multirow{2}{*}{ A. albopictus } & 우 & $39 \%$ & $33 \%$ & $59 \%$ \\
\hline & 占 & $50 \%$ & $89 \%$ & $67 \%$ \\
\hline \multirow{2}{*}{ A. aegypti } & 우 & $28 \%$ & $72 \%$ & $56 \%$ \\
\hline & $\hat{s}$ & $33 \%$ & $89 \%$ & $72 \%$ \\
\hline \multirow{2}{*}{ C.pipien spallens } & 우 & $39 \%$ & $67 \%$ & $71 \%$ \\
\hline & $\hat{o}$ & $22 \%$ & $83 \%$ & $58 \%$ \\
\hline C.pipiens & 우 & $44 \%$ & $61 \%$ & $55 \%$ \\
\hline quinquefasciatus & $\hat{\delta}$ & $28 \%$ & $83 \%$ & $48 \%$ \\
\hline C. pipiens molestus & 우 & $33 \%$ & $77 \%$ & $52 \%$ \\
\hline \multicolumn{2}{|l|}{ Average accuracy } & $35.11 \%$ & $72.67 \%$ & $59.77 \%$ \\
\hline
\end{tabular}

\section{DISCUSSION}

The result of automated identification was $72.67 \%$ average accurate by building the artificial neural network with the wingbeat frequency. As a characteristic, the wingbeat waveform alone was not very useful to automate identification with $35.11 \%$ average accurate by constructing the artificial neural network since all these wingbeat waveforms were very similar sine waves, it was probably the reason of un-efficient identification. The result directly demonstrated that it was feasible to identify wingbeat frequencies of mosquitoes by artificial neural network, while wingbeat waveform was not efficient. Meanwhile the research validate that Roth (1948) observed the sexual behavior of mosquitoes, which had been generally accepted that in most species the fundamental frequency of the flight sound of the female attracts males from far distances (Gillett, 1971).

Moore (2002) built artificial neural network to automated identification wingbeat waveform of aphids with $67 \%$ average accurate arrange from $41 \%$ to $94 \%$, the mean reasons included that there was no information communication between different species of aphids, it was more important that flying of aphids was in-continuous. Farmery (1981) showed that the wingbeat frequency stayed constant, the harmonic pattern of optically sensed wingbeat waveforms changes radically when the orientation of the insect with respect to the sensor was varied. So there were still many interesting fields to research on automated identification of species of insects in the future. 


\section{ACKNOWLEDGEMENTS}

This research was granted by the National Priority Program of Innovative Technology (863) No. 2002AA243031 and 2003AA2090. The author would like to extent sincere appreciation professor $\mathrm{Yu}$ yixin and professor $\mathrm{Gu}$ tao for their constructive advice.

\section{REFERENCES}

[1] Christophers S. R. Aedes aegypti(L). The yellow fever mosquito: its life

history, bionomic and structure .Cambridge University Press, London and New York. 1960, $739 \mathrm{pp}$

[2] Clements A. N. The physiology of mosquitoes. Pergamon Press, Oxord. 1963, 393pp

[3] David Chesmore. Automated Bioacoustic Identification of species. Annals of the Brazilian Academy of Sciences, 2004, 76(2): 435 440

[4] Do M.T, el al. A test of a pattern recognition system for identification of

spiders. Bulletin of Entomological Research, 1999, 89:217 224

[5] Farmery M.J. Optical studies of insect flight at low altitude. Ph. D. dissertation. York University, York, UK. 1981

[6] Gillett J. D. Mosquitoes Weidenfeild and Nicholson. London. 1971, 274pp

[7] Moore A. Development of a data acquisition system for long-term outdoor recording of insect flight activity using a photosensor. 13th Conference on Acrobiology and Biometeorology, American Meteorological Society, Albuquerque, New Mexico.1998

[8] Moore A, el al. Automated identification of flying insects by analysis of wingbeat frequencies. J. Econ. Entomol. 1986, 79: 1703 1706

[9] Moore A, el al. Automated Identification of Optically Sensed Aphid (Homoptera: Aphidae) Wingbeat Waveforms. J. Econ. Entomol. 2002, 95(1): 1 8

[10] Qubit Systems. WfRec Transient Waveform Recorder. Kingston, Ontario, Canada. 2001

[11] Peter Belton, el al. Flight sounds of the females of some mosquitoes of western Canada. Ent. Exp. \& appl. 1979, 26:105 114

[12] Parsons S, el al. Acoustic identification of twelve species of echolocating bat by discriminate function analysis and artificial neural networks. J. Exp. Bio. 2000, 203: 2641 2656.

[13] Roth L.M. A study of mosquito behavior. An experimental laboratory study of the sexual behaviour of Aedesaegypti(L).Amer. Mid. Nat. 1948, 40:265 352

[14] Taylor B. W, el al. Laboratory mating of Addes vexans (Diptera: Culicidae). Ann. Ent. Soc. Amer. 1974, 67: 137 138

[15] Williams C.M, el al. Oscilloscopic and stroboscopic analysis of the flight sounds of Drosophila Bol. Bull. (Wood's Hole) 1950, 99:300 307 